\title{
Energy Informatics Can Optimize the Design of Supply and Demand Networks
}

\author{
Robert Bradshaw ${ }^{1}$ and Brian Donnellan ${ }^{2}$ \\ ${ }^{1}$ National University of Ireland Maynooth, Maynooth, Ireland \\ ${ }^{2}$ National University of Ireland Maynooth, Innovation Value Institute, Maynooth, Ireland
}

Keywords: Green IS, Supply and Demand, Networks, Sustainability, Energy Informatics, Bikeshare.

Abstract: This paper proposes that a new green IS framework - Energy Informatics - may provide the best means of
optimising the design of supply and demand networks. The framework proposes an integrated systems
solution which incorporates technical and architectural design elements, eco-goals, and human stakeholders
and places a particular focus on the role of information systems in effectively integrating and managing
service supplier and service user information to optimize network efficiency. The paper explores the
potential of the framework through a case study of an innovative bikeshare initiate from MIT called The
Copenhagen Wheel. The study demonstrates that the framework has the potential to inform system design in
the bikeshare domain. Further research will be required to determine its potential in informing other supply
and demand areas.

\section{INTRODUCTION}

\subsection{Background}

A recurring theme in the sustainability literature is our continued reliance on the burning of fossil fuels for energy production. The consequence of this has been a significant increase in atmospheric carbon dioxide which in turn has resulted in a range of problems including air pollution, ocean acidification and a loss of biodiversity (Jacobson, 2008). A broad consensus exists amongst scientists and social commentators alike that reducing our levels of $\mathrm{CO}_{2}$ will be pivotal in addressing these problems. The response from businesses and corporations in particular, given their importance within our societies is increasingly seen as key to the success of the sustainability agenda. Due to the close attention of the media, lobbyists, and an increasingly ecoconscious public, all areas of corporate activity have now come under scrutiny and businesses are expected to be far more proactive, and indeed creative, in how they meet their sustainability obligations.

The information systems community has responded to this changing climate by exploring the potential of technology to work in conjunction with people, processes and business practices to deliver holistic solutions that can make entire systems more sustainable. This approach has become known generically as green IS or green information systems and it incorporates not only the principles of green information technology, which focuses largely on data centre and hardware efficiency, but also on the capacity of a range of digital tools, and information itself, to enable organisations and communities to become more sustainable. It recognises that sustainability is a multi-faceted concept involving economic, environmental and social contexts. While an information technology (IT) transmits, processes, or stores information, an information system (IS) is an integrated and cooperating set of people, processes, software, and information technologies to support individual, organizational, or social goals. The focus of this research is, therefore, on "green IS" rather than "green IT" because green IS gives us the potential to (i) measure and process vast amounts of data, (ii) transform physical processes into virtual ones and (iii) improve the efficiency of physical processes. Though very much in its infancy, the literature notes a role for green IS in a range of areas including traffic management systems, virtual presence technologies, and in supporting informed decision making in the provision and use of services. An increasingly important role for green IS is highlighted in the management of supply and demand networks. Supporting both service provision 
and service usage through intelligent systems design is an opportunity to fundamentally transform business processes while delivering on the economic, social and environmental imperatives of sustainability

\subsection{Energy Informatics}

Energy informatics (Watson et al, 2010) is an IS framework which specifically addresses this area. The framework proposes an integrated approach to the design and implementation of systems which support energy efficiency while adopting specific architecture and design elements. The core concept behind the energy informatics (EI) approach is that energy + information should result in the consumption of less energy. As such, the framework concerns itself with improving the efficiency of energy demand and supply systems. The framework aims to incorporate disciplines such as management science, design science and policy formation in conjunction with high granular data about the provision and use of energy to develop systems that can improve outcomes for the environment. The framework is illustrated below.

- Suppliers can more effectively manage service delivery if supplied with the appropriate usage information from the consumer (Watson et al, 2009)

A flow network is a set of interconnected transport elements that enables the movement of continuous matter such as oil, electricity, water etc or discrete objects such as cars, bikes, packages or people (Watson et al, 2010). A sensor network, as defined by the energy informatics framework, is a set of connected, distributed devices whose purpose is to report on the status of some physical object or environmental condition i.e. air pollution or machine health (Milenkovic et al, 2006). Effective sensor networks are reliant on fine grained information. A sensitized object is a physical item which is owned by the energy consumer and has the ability to gather and report data about its use. Domestic appliances for example can be sensitized with smart plugs which report on power usage and support smart metering (Jahn, 2010). Of central importance to the framework is the capacity of sensitized objects to give the consumer the information they need to use the object intelligently and with the environment in mind. The role of the information system is to integrate all the other elements of the framework to provide a complete design. The technical elements of the framework are augmented by integrating social and organisational contexts, eco-goals and the primary stakeholders in the supply and demand network paradigm i.e. service suppliers and service users.

Eco-goals are well documented in the sustainability literature. Unsurprisingly, cost saving remains high on the list of expected returns from green IS investment. Corporations are largely motivated to pursue eco-efficiency by the prospect of cost reduction and it typically involves the "efficient" use of resources in order to reduce negative impacts on the environment (Dedrick, 2010). Eco-equity relates to the principle that all peoples and generations should have equal rights to environmental resources (Gray, Bebbington, 2000).

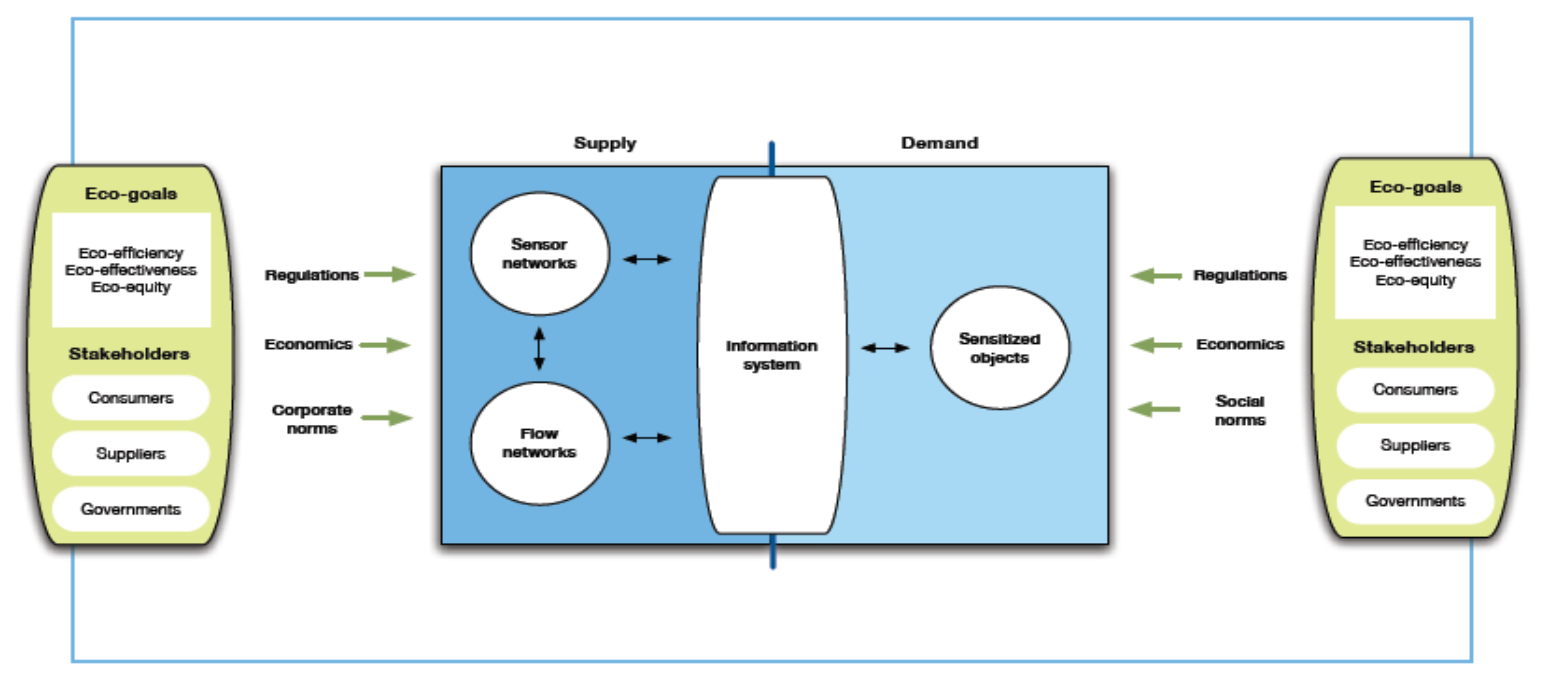

Figure 1: Energy Informatics Framework (Watson et al, 2010). 
Developing these norms and ensuring that energy sustainability is seen as an imperative will require the active support of opinion leaders and governments (Watson et al 2010). Eco-effectiveness is concerned with "doing the right things" as opposed to "doing things right". Political leaders in Denmark for instance have levied a $180 \%$ tax on petrol engined cars while zero-emission vehicles are exempt, and the New York Times (29 July, 2011) notes that countries such as France, Germany, Britain, Portugal and Spain all heavily subsidize the purchase of electric vehicles (EVs). Similar approaches are in evidence in both the US and Asia (Ahman, 2004).

In addition Watson proposes four key design elements. These are ubiquity, uniqueness, unison and universality and their usefulness in the design of information systems is well established within the IS literature (Outram, 2010, Tzeng, 2008, Sammer, 2011, Galanxhi-Janaqi, 2004, Placido et al, 2011). Ubiquity is access "to information unconstrained by time and space" (Junglas, Watson, 2006). Providing ubiquitous access to information about a service enables users to access information from wherever they may be located and to explore their options to increase the usefulness of that service. Unison, sometimes referred to as consistency, proposes that the procedure of accessing information varies as little as possible. This might mean that users could access information from multiple services or locations while needing only to learn a single procedure. Universality relates to the drive to reduce compatibility issues or friction between information systems in order to achieve seamless data exchange. XML (extensible markup language), web services, and application programming interfaces (APIs) have become the de facto means of achieving this interoperability (Rainer and Cegielski, 2011, pp184). Uniqueness is described in the literature as "knowing precisely the characteristics and location of a person or entity" (Junglas, Watson, 2006). With information, it can be used to find the best match between the user's needs and the physical resources available. Many bikesharing schemes for example uniquely identify both bikes and users, which means that users can view the availability of bikes and parking spaces on a station by station basis while system administrators can use usage patterns to inform fleet management and other operational functions (Buttner et al, 2012)

Research suggests, (Watson, 2009, Outram et al 2010, Midgely 2009, Chowdhury, 2007), that the more successful systems have supported their physical infrastructure with information systems which implement these elements. They "minimise the limitations of the physical system and enable and support users to adopt behaviours that help rather than hinder the environment" (Outram et al, 2010).

\section{EI IN PRACTICE - THE COPENHAGEN WHEEL}

Bikeshare schemes have become an increasingly popular phenomenon in recent years as urban planners across the world have used them to improve urban mobility and reduce the environmental impact of motorised transportation systems. The basic premise of the schemes is that bikes are made available throughout the city environment and are then used to support what are, for the most part, relatively short trips. The schemes have the added benefit of providing a link between existing transport nodes and required destinations. System providers include governments, publicprivate partnerships, transport agencies, universities, advertising agencies and for-profit organisations (Midgely, 2011). Schemes typically use independent docking stations capable of automatically checkingout and returning bikes. Users are required to subscribe to the schemes initially and can then access the bikes through a variety of technologies which include smart cards, fobs, direct access codes, or SMS (Buttner et al, 2011). System information, such as the availability of free bikes and stands, or the riders' usage statistics, is typically made available through web based applications, or at interfaces incorporated into the station kiosks. Kiosks can usually support registration and payment options. The recent adoption of mobile phone applications by many schemes has also improved access and usability (Buttner et al, 2011). From an EI perspective, bikeshare schemes can be seen to represent conventional supply and demand networks which attempt to manage the "flow" of bikes in order to maximise the number of trips.

The Copenhagen wheel is a research project developed in 2009 by the Massachusetts Institute of Technology's SENSEable City lab for the Kobenhavns Kommune - the Copenhagen Municipality. Though not being developed exclusively for the bikeshare environment, the development team anticipate that this will be its primary application. Through the use of mobile and web technologies the wheel attempts to replace the traditional kiosk and docking station model and allow bikes to be secured to any traditional bike 
rack. This stationless design requires significantly reduced infrastructure and investment. To enhance usability and appeal to a greater cross section of users, the bikes are electric hybrid vehicles. The rear wheel hub, using a Kinetic Energy Recovery System (KERS), captures the dissipated energy from pedalling and braking using torque sensors and stores it until needed by the rider. The hub also contains real-time sensing technology which can transmit information about the environment, personal fitness and location to an integrated smartphone and also via the cell phone network to the web. The sensitized object in this design is represented by the hub.

The sensors it contains can detect $\mathrm{CO}_{2}$, NOx (fig $2)$, temperature, noise $(\mathrm{dB})$ and humidity. In addition to environmental sensing, the suite also monitors the riders speed, relative inclination (through the use of a gyroscope), distances travelled and so on. See figure 3 .

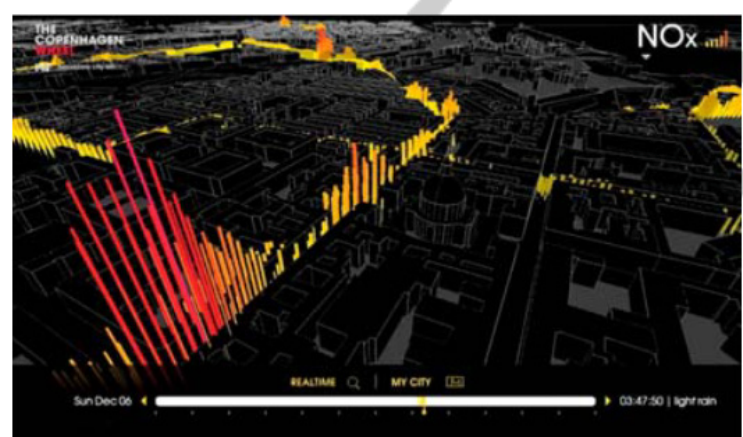

Figure 2: NOx data collected while cycling in Copenhagen during December 2009 (Outram, 2010).

The system uses GPS to provide its sensor network which supports active tracking. Route information, plus the data generated locally by the sensor suite, is then relayed to a central server using the cellular network. This allows riders to review the data in their own time using the systems' web based interface. Data developed over extended time periods can also be used to support predictive and demand modelling. In addition, the real-time visibility of the bikes significantly reduces the impact of theft and vandalism.

The information system in the Copenhagen Wheel is an open, XML compliant platform and designed with high levels of inter-operability and universality in mind. The scheme provides social media functionality within its site to support user interaction and has also developed links to Facebook and Google+. Riders see any badges or awards they or their friends have received from the system for achieving personal targets in relation to calories burned or $\mathrm{CO}_{2}$ offset etc. Using social media to support information exchange also encourages riders to develop relationships which can improve safety from both physical and psychological perspectives. In addition, the scheme recognises the importance of incorporating external data sets which have the potential to enhance usability and performance. An app to incorporate real-time weather forecast data into the existing information suite is currently being deployed for example and the developers are also focused on incorporating data from other transit modes. This should enable enhanced trip planning and customisation across public transportation systems and/or car-to-go schemes.

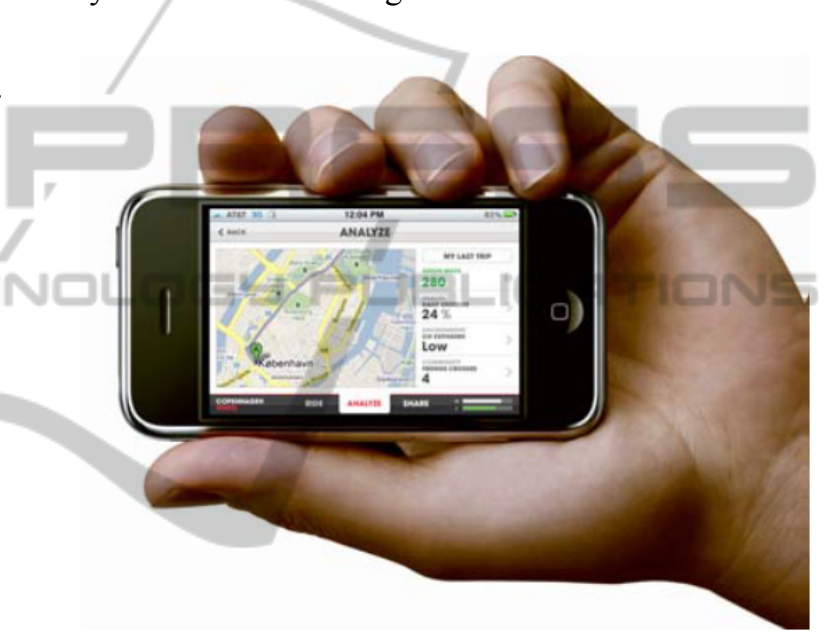

Figure 3: Smartphone analyser showing environmental and health data.

Unison or information consistency is supported by a common system view which is provided by an integrated database and uniqueness is enabled by having visibility of riders throughout the entirety of the usage period. Visibility of the bikes at all times enables accurate systems updates to be provided to riders and supports meaningful bike distribution by system operators. Ubiquity is perhaps the schemes' defining characteristic. Multiple streams of real-time data are available to riders at all times via the smartphone interface which mirrors the trend towards the use of dashboard telemetry in motorised transportation as a way of informing driver behaviour. To avoid cognitive overload the rider can choose to view as much or as little data as they feel appropriate.

The Copenhagen Wheel places a high value on reciprocal relationships, both internal and external. Internally, the scheme encourages riders, through the use of incentives, to add value to the data collected 
through feedback and route annotation. This user generated content can be seen as an additional sensor network complimenting and supporting the scheme's sensing technologies. Externally, the Copenhagen Wheel is focused on creating partnerships with both local government and independent $3^{\text {rd }}$ parties. Through these relationships the value of the data collected can be properly exploited i.e. it can inform urban planning, drive improvements in cycling infrastructure or enhance integration with other transportation modes.

Figure 4 illustrates the Copenhagen Wheel from an EI perspective.

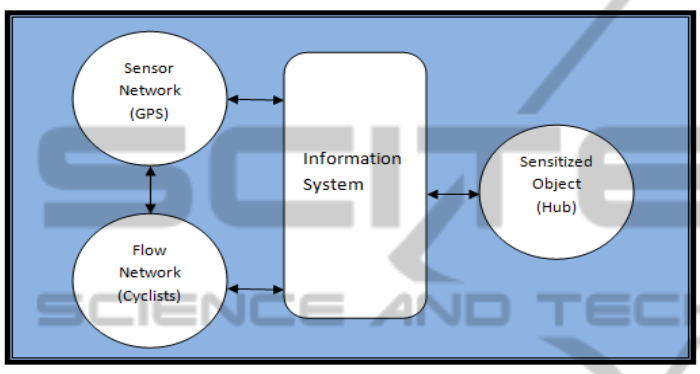

Figure 4: Energy Informatics and the Copenhagen Wheel.

\section{CONCLUSIONS}

\subsection{Addressing the Initial Position}

The effectiveness of system design, i.e. the degree to which both sides of the supply and demand paradigm are supported, can be seen to be a function of the characteristics of the information being disseminated within the scheme. The variety, reliability, timeliness and granularity of information "sensed" by the combination of sensitized object and sensor network, and distributed by the central information system, have a direct bearing on the degree of visibility providers have of system usage which in turn impacts on a wide range of operational and management functions. These include bikedistribution, fleet maintenance, infrastructure planning, and the management of threats such as theft and vandalism. Additional information streams, imported from the external environment have the potential to enhance overall system performance and improve the level of connectedness schemes have with their environments. Providing accurate, granular data on how riders are using the system allows them to make informed choices about their behaviour.

In effect, the right information can support the dynamic adjustment of supply and demand requirements. "Flow", in the form of bikes and riders, can be optimised by the use of smart technologies and digital tools combined in an architecture which integrates key system stakeholders. These are the core principles of the EI framework. The framework does not prescribe a particular set of technologies or architectures per se but instead proposes a set of principles by which the potential of information can be leveraged to optimise efficiency. It provides an understanding of the role played by each of the components that comprise the overall supply and demand network and provides a blueprint for exploiting them to optimise performance.

The contextual deminsions of energy informatics are also supported by the case study. It demonstrates that eco-goals, an important element in Watson's framework, can be major contributors to design and performance. It illustrates for example that ecoefficiency and eco-effectiveness need not be mutually exclusive. On the contrary, it suggests that the stationless design, which impacts least on its environment and supports the greatest levels of usability and customisation, is also the most cost effective. In summary, improved design is achieved by:

- Allowing schemes to be understood from the perspective of supply and demand networks.

- Providing a frame of reference by which the value of their existing information and technical infrastructure can be understood and evaluated.

- Recommending a set of informational attributes, design elements and eco-goals which can be used to improve performance and sustainability.

\subsection{Future Research Opportunities}

Useful further research would be to explore the potential of the framework to support the design of networks across other domains. In addition to the transportation environment, opportunities exist for example in areas such as the delivery of services such as electricity, water, or gas. These environments represent more conventional supply and demand relationships yet the potential of information to support both service provision and service use in a manner similar to bikesharing is high and the industries are influenced by regulatory and environmental factors that also resonate with the bikesharing domain i.e. political stakeholders, corporate motivations and so on.

There may also be potential in exploring the 
value of informational tools in increasing the efficiency of areas such as open data platforms, telephony or internet service provision, which again contain the core elements of supply and demand networks i.e. service providers, service users, and a flow of content to be regulated. It would also be interesting for instance to establish to what degree collaborative relationships impact the design of networks in these environments.

\section{REFERENCES}

Ahman, M (2004) Government policy and the development of electric vehicles in Japan - Energy Policy 34 (2006) 433-443 Science Direct [Accessed 11/01/2012]

Buttner, J. Peterson, T. Reth, P (2011) Optimising Bikesharing in European Cities (OBIS): A Handbook http://www.obisproject.com/ - [Accessed 17/12/2011]

Chowdhury, G. (2011) Building Environmentally Sustainable Information Services: A Green IS Research Agenda. Journal of the American Society for Information Science and Technology Volume 63, Issue 4, pp 633-647 Wiley Online [Accessed $12 / 06 / 2012]$

Dedrick, J. (2010) Green IS: Concepts and Issues for Information Systems Research. Communications of the Association for Information Systems Vol 27 No1 [Accessed 15/2/2012]

Gray, R. Bebbington, J. (2000) Environmental Accounting, Managerialism and Sustainability: Is the Planet Safe in the Hands of Business and Accounting? Advances in Environmental Accounting and Management Vol 1 No 1 pp. 1-44. Emerald Database [Accessed 09/01/2012]

Jacobson, M (2008) Geophysical Research Letters Vol. 35, L03809, doi:10.1029/2007GL031101, http://cleanairinitiative.org/portal/system/files/articles72482_full_0.pdf [Accessed 1/6/2012]

Jahn, M. Jentsch, M. Prause, C.R. Pramudianto, F. AlAkkad, A. Reiners, R. (2010) The Energy Aware Smart Home - Future Information Technology (FutureTech), 2010 5th International Conference on IEEEXplore Digital Library - [Accessed 4/01/2012]

Junglas, I. Watson, R. (2006) The U-constructs: Four Information Drives. Communications of AIS 17, 569592. Issue 17, p2-43, 42p EBSCO [Accessed 13/01/2012]

Midgely, P (2011) Bicycle-Sharing Schemes: Enhancing Sustainable Mobility in Urban Areas https://www.un.org/esa/dsd/resources/res_pdfs/csd19/Background-Paper8-P.Midgley-Bicycle.pdf. [Accessed 19/12/2011]

Milenkovic, A. Otto, C. Jovanov, E. (2006) Wireless sensor Networks for Personal Health Monitoring. Computer Communications Vol 13-14, pp 2521-2533 SciVerse database [accessed 24/06/2012]
Outram, C. Ratti, C. Biderman, A (2010) The Copenhagen Wheel: An Innovative Electric Bicycle System that Harnesses the Power of Real-Time Information and Crowd Sourcing - http://cgt.columbia.edu/files/ papers/Outram_Ratti_Biderman_EVER2010_Monaco. pdf [Accessed 14/01/2012]

Rainer, K. Cegielski, C. (2010) Introduction to Information Systems Enabling and Transforming Business $3^{\text {rd }}$ Edition Wiley

Sammer, T. Carmicael, F. Andrea, B (2011) Integrating the Smartphone: Applying the U-Constructs Approach on the Case of University Mobile Services. http://www.slideshare.net/thfs/mcis2011-integratingthe-smartphone-applying-the-uconstructs-approachon-the-case-of-mobile-university-services [Accessed $12 / 05 / 2012]$

Tzeng, S. Chen, W. Pai, F (2008) Evaluating the business value of RFID: Evidence from Five Case Studies. Int. J. Production Economics 112 (2008) 601-613 SciVerse database [Accessed 12/06/2012]

Watson, R Boudreau, M (2009) Energy Informatics Driving Change Through Competitive Thought Leadership SIM Advanced Practices Council [Accessed 17/12/2011]

Watson, R. Boudreau, M. Chen, A. (2010) Information Systems and Environmentally Sustainable Development: Energy Informatics and New Directions for the Community. MIS Quarterly Vol. 34 No 1, pp 23-38

Wood, G. Newborough, M (2002) Dynamic EnergyConsumption Indicators for Domestic Appliances: Environment, Behaviour and Design Energy and Buildings, Volume 35, Issue 8, September 2003, Pages 821-841 Science Direct [2/01/2012] 